\title{
CORRIGENDUM
}

\section{Connective tissue growth factor modulates oral squamous cell carcinoma invasion by activating a miR-504/FOXP1 signalling}

M-H Yang, B-R Lin, C-H Chang, S-T Chen, S-K Lin, MY-P Kuo, Y-M Jeng, M-L Kuo and C-C Chang

Oncogene (2013) 32, 670; doi:10.1038/onc.2012.559

Correction to: Oncogene (2012) 31, 2401-2411; doi:10.1038/ onc.2011.423; published online 19 September 2011

In this paper the Methods section describing the microarray analysis and the GSE number is incorrect owing to the initially unsuccessful uploading of microarray data set and typesetting error. We re-upload the original microarray raw data and the description in Methods section should be 'lllumina human V2 microRNA expression beadchip was used for microRNA expression analysis. The raw data of the spot density were extracted from the Illumina BeadStudio software and deposited into the Gene Expression Omnibus (GEO) database (accession number GSE41845)'. We thank Dr Kenneth W Witwer (The Johns Hopkins University School of Medicine) for pointing out this error.

The authors sincerely apologize for the error, and regret any confusion and/or inconvenience it may have caused. 